\title{
In Situ Biasing of Tapered Si-Ge NW Heterojunctions using Off-Axis Electron Holography
}

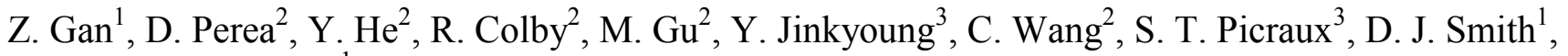 \\ and M. R. McCartney ${ }^{1}$ \\ 1. Department of Physics, Arizona State University, Tempe, AZ 85287. \\ 2. Environmental Molecular Sciences Laboratory, Pacific Northwest National Laboratory, Richland, WA \\ 99352. \\ 3. Center for Integrated Nanotechnologies, Los Alamos National Laboratory, Los Alamos, NM 87545.
}

Heterojunction Si-Ge nanowires (NWs) have potential applications such as field effect transistors [1]. Knowledge of the active dopant concentration and distribution, and resultant built-in potential across the $\mathrm{Si}-\mathrm{Ge} p-n$ junction under biasing conditions is important for improving device performance. Off-axis electron holography is an effective method to measure electrostatic potential with nanoscale resolution $[2,3]$. By reconstructing phase images from interference holograms, the projected potential distribution of the sample along the electron beam direction can be quantitatively mapped. Here, we have used off-axis electron holography to measure the potential profile across $p$ - $n$ heterojunctions in Si-Ge NWs in situ under varying biasing conditions. The heterojunction Si-Ge NWs are grown using the vapor-liquid-solid method with AuGa catalyst [4], and consist of a B-doped $\left(\sim 10^{18} \mathrm{~cm}^{-3}\right)$ tapered Ge base and an untapered P-doped $\left(\sim 10^{19} \mathrm{~cm}^{-3}\right)$ Si tip.

Figure 1a and $\mathrm{b}$ show TEM and HAADF-STEM images, respectively, of a typical Si-Ge NW where the Ge portion is tapered due to vapor-solid surface growth. Some small particles are observed on the NW surface most likely due to catalyst breakup when changing growth temperature and reactant gases from $\mathrm{Ge}$ to Si. Figure 2 shows the hologram of a typical unbiased Si-Ge NW, while the resultant reconstructed phase image is shown in Figure 3a. The Ge region has higher phase due to its higher mean inner potential (MIP) and greater thickness. A slightly higher phase is also observed for Si near the catalyst. The reason is not yet clear but may be due to higher dopant concentration. After accounting for the thickness and electron interaction factor, the total potential profile can be extracted as shown in Figure 3b. The potential goes from 13.5 V in Ge to $11.7 \mathrm{~V}$ in Si. Since the MIP is $14.3 \mathrm{~V}$ for Ge and 12.1 $\mathrm{V}$ for $\mathrm{Si}$, then the built-in potential is $0.4 \mathrm{~V}$ with the $\mathrm{Ge}$ side being lower. Using the dopant concentrations estimated from the growth conditions, the built-in potential is calculated to be $0.43 \mathrm{~V}$. Thus, the holography result is consistent, and suggests that most of the dopants are active. The Si-Ge NW was then connected via tungsten contacts to an in situ biasing TEM holder with Si biased and Ge grounded. The I-V curve measured in Figure 4a indicates rectifying behavior. In forward bias, turn-on is observed above $\sim 1 \mathrm{~V}$. On the other hand, in reverse bias, the NW has higher resistance and because of tunneling, there is still current through the junction. Holograms were taken under varying biasing conditions and the measured phase and potential line profiles are shown in figure $4 \mathrm{~b}$. Under positive bias, the junction is in reverse bias and the junction resistance is high with most of the voltage across the junction. Under negative bias, the junction is in forward bias and the junction resistance is low, so that not all of the voltage is applied to the junction and the change of phase and potential is small. Further simulations are in progress to interpret these biasing results [5].

\section{References:}

[1] A. M. Ionescu and H. Riel, Nature 479 (2011) 329. 
[2] M. R. McCartney and D. J. Smith, Ann. Rev. Mater. Res. 37 (2007) 729.

[3] Z.Gan, et al., Applied Physics Letters, 103 (2013) 153108. [4] D. Perea, et al., Nano Lett, 11 (2011) 3117.

[5] The electron holography studies have been supported by DoE Grant DE-FG02-04ER46168. We gratefully acknowledge the use of facilities within HREM center at Arizona State University. We also acknowledge use of the CINT and EMSL user facilities.
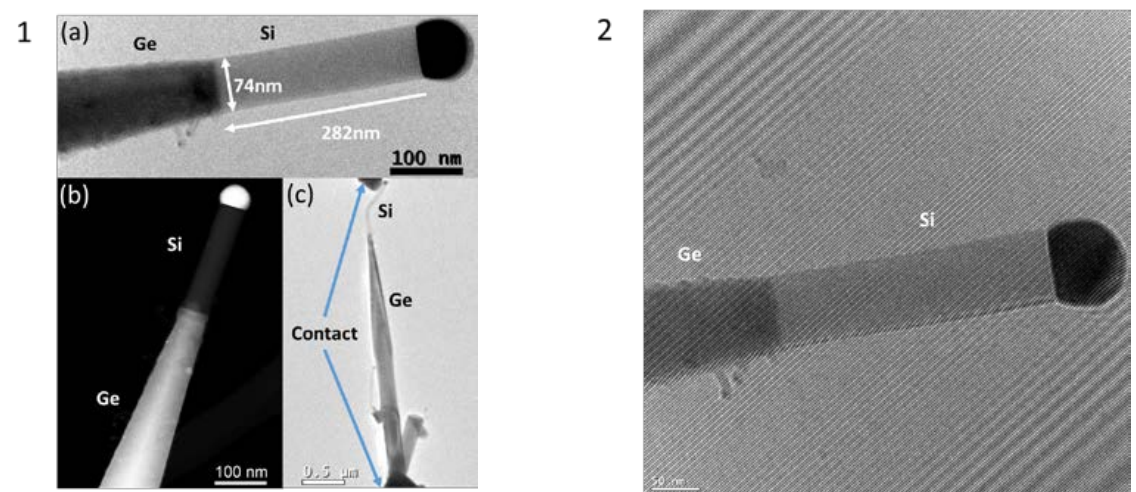

Figure 1. (a) TEM image, and (b) HAADF-STEM image of Si-Ge NW. (c) TEM image of Si-Ge NW mounted to tungsten contacts. Figure 2. Hologram of typical Si-Ge NW without bias.
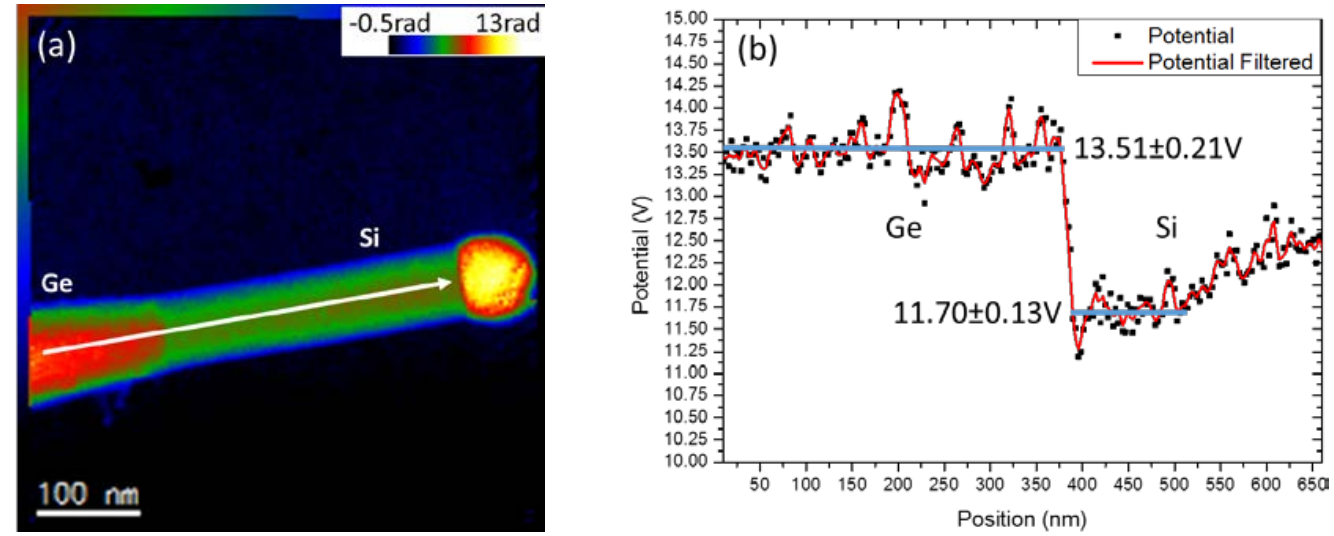

Figure 3. (a) Reconstructed phase image of Si-Ge NW in pseudo-color without bias. (b) Measured potential line profile along white arrow in (a).

Figure 4. (a) Measured I-V curve. The bias is on Si (n) side. (b) Phase and potential line profiles measured under bias. The change of thickness is not considered. 